\title{
Thermal fatigue of electrical fuses
}

\author{
Jean-Louis Gelet
}

MERSEN France SB SAS, 15 rue Jacques de Vaucanson, 69720 Saint-Bonnet de Mure, France

\begin{abstract}
Electric Fuses have to respect different national or international standards such as IEC (International Electro-technical Commission) 269. These standards define the characteristics of the fuses and describe the tests to be run in order to check fuse's ability to take up their main functions, i.e. current-conduction and operation under overloads and short-circuits. But fuses never carry current neither operate under standardized conditions. For example, rated current is evaluated under specified ambient temperature, without cooling air-flow, and with 1 meter-long connection-cables on both sides. In the field, temperature can reach up $80^{\circ} \mathrm{C}$, with or without air-flow and connection-parts are much more shorter. An issue is that current is never constant, often being cyclingly applied; equipments are frequently in use during the day and stopped in the night. ON-time and OFF-time generate alternative heating, then alternative stresses leading to thermal fatigue. MERSEN run many tests along the years, allowing to develop a method for choosing right fuses for each application. As a result, fuses don't melt unexpectedly in the field, but the method is supposed to be conservative and does not permit to get a better understanding of the phenomena neither an improvement of the products. The paper presents some specific ageing-tests run on conductive elements and tries to establish a correspondence between these tests and others carried out on complete fuses. Tests have been run on silver and copper, but their principle could be interesting for any structural material, especially because it underlines crack-opening.
\end{abstract}

\section{Basic phenomena}

When fuses are used under cycling conditions, alternation of stress and relaxation lead to some ageing by thermal fatigue.

Electrical, thermal and mechanical phenomena are chaining as follows:

1. Electrical current brings the fuse-element conductor to heat,

2. There is a tendency for the heated fuse-element to expand,

3. Thermal expansion is impeded by a sand-core around the conductor,

4. Stresses are then applied all along the conductor,

5. Stresses are transmitted and amplified at the notches,

6. When current stops, stresses are relaxed and even reversed in same cases,

7. Repetition of stress + relaxation leads to metallurgical fatigue of the fuse-element.

This is an Open Access article distributed under the terms of the Creative Commons Attribution License 4.0, which permits unrestricted use, distribution, and reproduction in any medium, provided the original work is properly cited. 


\section{MATEC Web of Conferences}
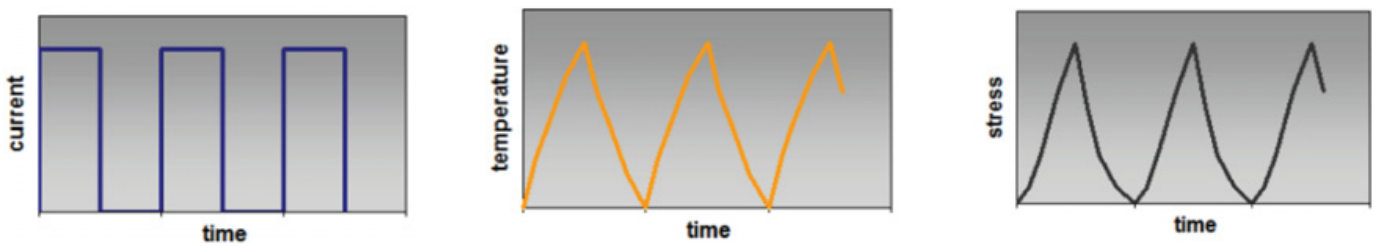

Figure 1. Curves of the 3 main phenomena involved in fuse-ageing vs. time.

Table 1. Number of cycles according to stress-level.

\begin{tabular}{|c|c|c|}
\hline $\begin{array}{c}\text { the level of stresses is over } \\
\text { the yield strength of the } \\
\text { conducting metal }\end{array}$ & $\begin{array}{c}\text { then occurs a plastic, so } \\
\text { called oligocyclic fatigue } \\
\text { (oligo = a few), }\end{array}$ & $\begin{array}{c}\text { low number of cycles } \\
\left(\leq 10^{4} \text { cycles) }\right.\end{array}$ \\
\hline $\begin{array}{c}\text { the level of stresses is lower } \\
\text { then the yield strength }\end{array}$ & then occurs a elastic fatigue & $\begin{array}{c}\text { number of cycles increases a } \\
\text { lot before rupture } \\
\text { (melting in case of a fuse) }\end{array}$ \\
\hline level of stresses is very low & no rupture will occur & $\begin{array}{c}\text { as an example, in case of } \\
\text { pure silver, } 2 \cdot 10^{6} \text { is usually } \\
\text { accepted as the number of } \\
\text { cycles for fatigue-endurance }\end{array}$ \\
\hline
\end{tabular}

\section{NT59 method}

Couplings between electrics, thermals, mechanics and theory of metallurgical fatigue - as described here-above-lead to complex considerations. On another hand, fuses are components of electro-technical industry, developed by electricians for electricians. It is more convenient for these engineers to run electrical tests than to go to model basic coupled phenomena.

NT59 is a code-designation for a dedicated method enable to predict life-duration of a fuse in an industrial application, or reversly to choose the good fuse for a specific equipment.

Using several hundreds of tests, Mersen's engineers have been able to draw out some behavioral laws. The electrical-stress ratio can be defined in different ways:

a) the stress corresponds to the global heating of the fuse. It is expressed as the ratio of the RMScurrent during the electrical cycle to the rated current of the fuse:

$$
\mathrm{A}=\mathrm{Ieff} / \mathrm{In}
$$

b) the stress corresponds to the heating of the notches. It is expressed as the ration of the actual current to the melting current under the conduction time:

$$
\mathrm{B}=\mathrm{I} / \text { Imelting }
$$

Electrical stress ratios (A or B) are subjected to be corrected by a set of coefficients:

For $\mathrm{t}_{\mathrm{ON}}=\mathrm{t}_{\mathrm{OFF}}=1$ hour, coefficient $\mathrm{k}$ is given to 1

If $\mathrm{t}_{\mathrm{ON}}$ and $\mathrm{t}_{\mathrm{OFF}}$ are larger than 1 hour, then $\mathrm{k}$ is kept to 1 .

If $t_{\mathrm{ON}}$ or $\mathrm{t}_{\mathrm{OFF}}$ is less than 1 hour, then the abacus gives the value of $\mathrm{k}$ to be applied. For examples:

$$
\begin{gathered}
t_{O N}=t_{O F F}=80 \mathrm{sec}, k=1.2 \\
t_{O N}=80 \mathrm{sec} \& t_{O F F}=15 \mathrm{sec}, k=1.3 .
\end{gathered}
$$




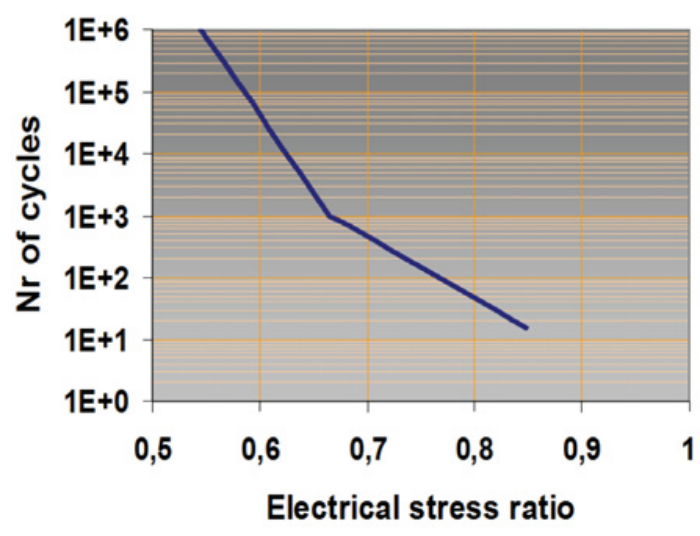

Figure 2. Numbers of cycles vs. electrical stress ratio (A or B).

Table 2. Correction coefficients to be applied to electrical stress ratios.

\begin{tabular}{|c|l|}
\hline A1 & temperature correction coefficient: takes into account ambient temperature \\
\hline Bv & air-blowing correction coefficient: takes into account the speed of blowing air on the fuse \\
\hline $\mathrm{C} 1$ & $\begin{array}{l}\text { connection correction coefficient the fuse never uses in the field with the same connections } \\
\text { (copper-bar sizes or cable-diameters) as during lab-tests; } \mathrm{C} 1 \text { allows to take into account the } \\
\text { connection-conditions in the field }\end{array}$ \\
\hline $\mathrm{Cpe}$ & $\begin{array}{l}\text { frequency correction coefficient takes into account skin and proximity effects due to high frequencies; } \\
\text { this effects begins to be significant at a few hundred of } \mathrm{Hz}\end{array}$ \\
\hline $\mathrm{k}$ & $\begin{array}{l}\text { cyclic ratio correction coefficient takes into account that } \mathrm{t}_{\mathrm{ON}} \text { and } \mathrm{t}_{\mathrm{OFF}} \text { may be different and shorter than } \\
\text { necessary time for thermal equilibrium }\end{array}$ \\
\hline
\end{tabular}

\section{Accelerated ageing tests}

Of course, it is not conceivable to run tests which would give same life-durations as in the field as these are required for 10 to 30 years.

At first, Mersen's engineers use to apply a 1 hour ON +1 hour OFF test. This test consists in applying a level of electrical current to the fuse for 1 hour, this current inducing the conduction-element to be heated. Then the current is turned off for 1 hour, the conducting element cooling down. It is generally estimated that 1 hour ON is long enough to go beyond thermal transient period. For Ultra-Fast Fuses, life-duration under 1 hour at the rated current In +1 hour OFF, will be about 3 to 400 cycles, i.e. 25 to 33 days.

The matter is that this kind of tests will not allow to get high number of cycles. Even within one year, only a few thousands of cycles will be reached, i.e. beneath the transition from plastic to elastic strains. Of course, shorter $\mathrm{t}_{\mathrm{ON}}$ and $\mathrm{t}_{\mathrm{OFF}}$ can be imagined. Actually Mersen carried out many tests under these conditions. They led to the k-coefficient-abacus. But they didn't permit to approach the fatigueendurance of the electrical conductor, which should be necessary for a vertical asymptote to the curve of the Fig. 2. For that reason, a specific mechanical flat bending tests machine has been designed.

This machine, operating at $3000 \mathrm{rpm}$, gave the opportunity to reach $10^{8}$ cycles in a time as short as 3 weeks. Unfortunately, acting phenomenon is far from the one in the field, mainly because they are run at room-temperature. Speed is also an effective parameter. 


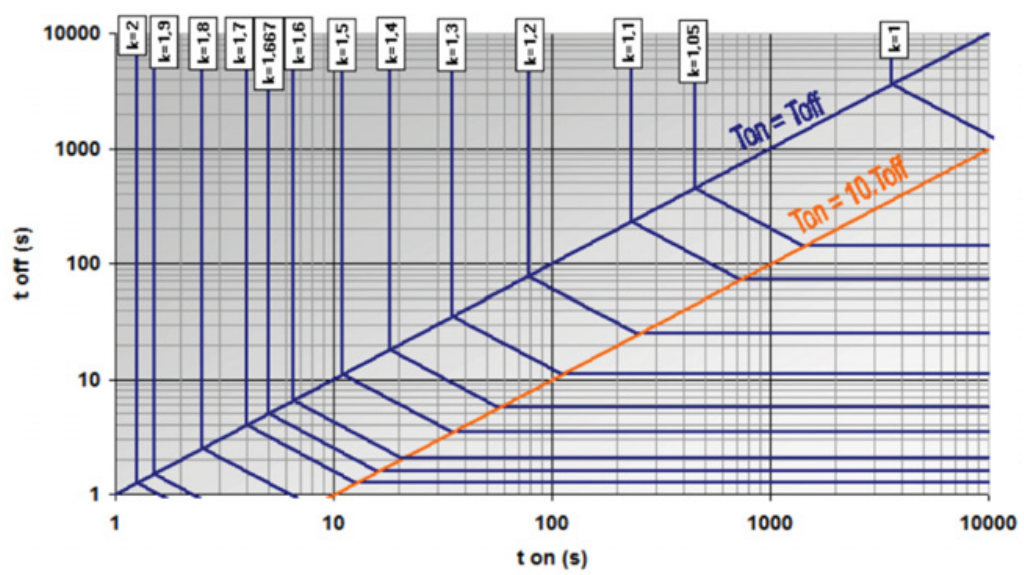

Figure 3. Abacus for k coefficient vs. $\mathrm{t}_{\mathrm{ON}}$ and $\mathrm{t}_{\mathrm{OFF}}$.

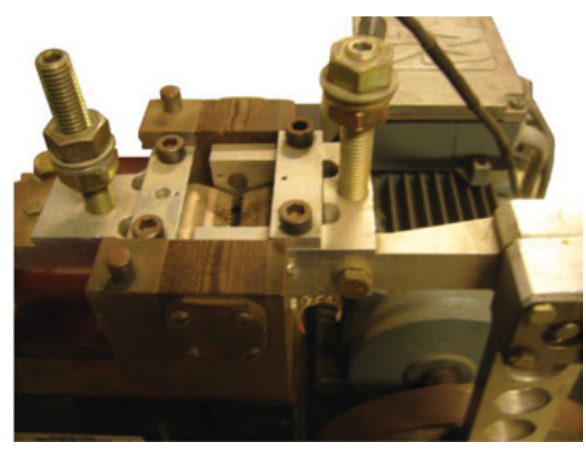

Figure 4. Mechanical flat bending test-machine.

\section{Dummy fuse with reduced thermal time constant}

A single fuse-element is placed on an aluminium cooling-water-box. An insulating tape does electrical insulation between fuse-element and water-box. In spite of this, there is a good thermal conductivity between the fuse-element and the cooling-box. Notches are surrounded by sand. The sand is held inside a fuse-ceramic-body. In addition, the drop-voltage between each side of the raw of notches is permanently measured. It allows to get the mean temperature of the notches from the variation of resistivity of the fuse-element-metal versus temperature.

$$
R_{\theta}=R_{0}(1+\alpha \cdot \theta)
$$

then:

$$
\theta=\frac{1}{\alpha}\left(\frac{\Delta U}{i}-1\right)
$$

Principle of the tests:

(1) fuse-element (2) notches (3) sand (4) ceramic body (5) insulating (6) water-box (7) drop-voltage

Thanks to this arrangement, it has been possible to reduce drastically the thermal time constant of sample and then to decrease the cycle-duration. Typically, tests have been run an-time $=10 \mathrm{sec}$ and OFF-time $=10 \mathrm{sec}$. That allowed to get tests up to $8 \cdot 10^{4}$ cycles. Also very interesting is the 


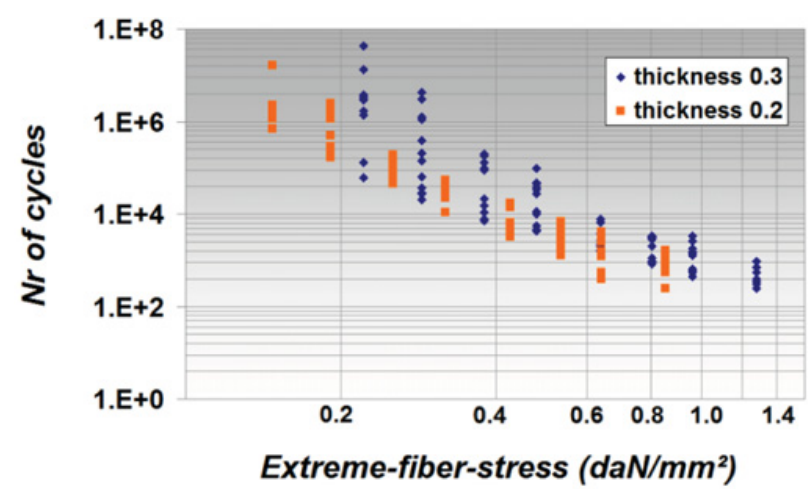

Figure 5. Mechanical flat bending test-results for Ag 99.995.

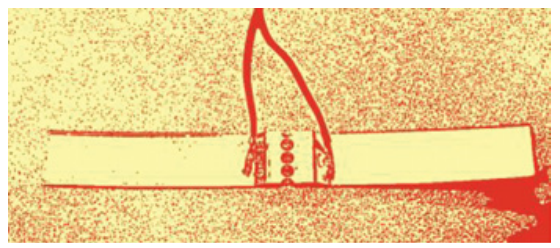

Figure 6. Fuse-element with 2 drop-voltage wires.

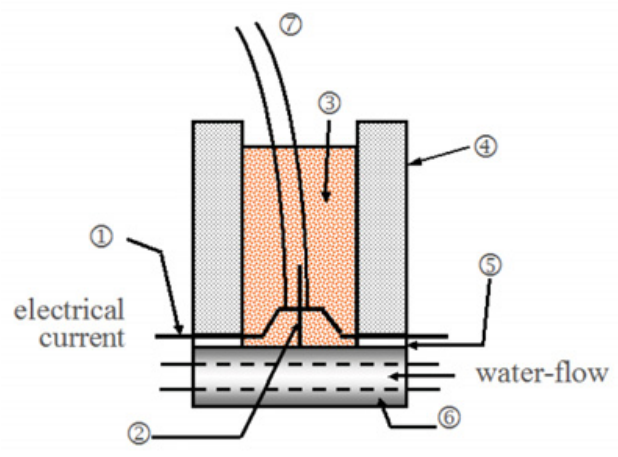

Figure 7. Schematic cross-section of the test-arrangement.

contribution of the voltage-drop-measurement. Beginning of crack-opening is detected as well as final rupture.

In order to draw out this curve, it has been considered that crack opened when an increase of the electrical resistance of $1 \%$ is detected. Then the following relationship is found:

$$
N_{\text {cycles_to_rupture }}=4.8 \cdot\left(N_{\text {cycles_for__ } 4 R=1 \%}\right)^{0.9}
$$

with a very good regression-coefficient of 0.976 . We will keep in mind that number of cycles to rupture is roughly 5 times larger that the number of cycles for $\Delta \mathrm{R}=1 \%$. 


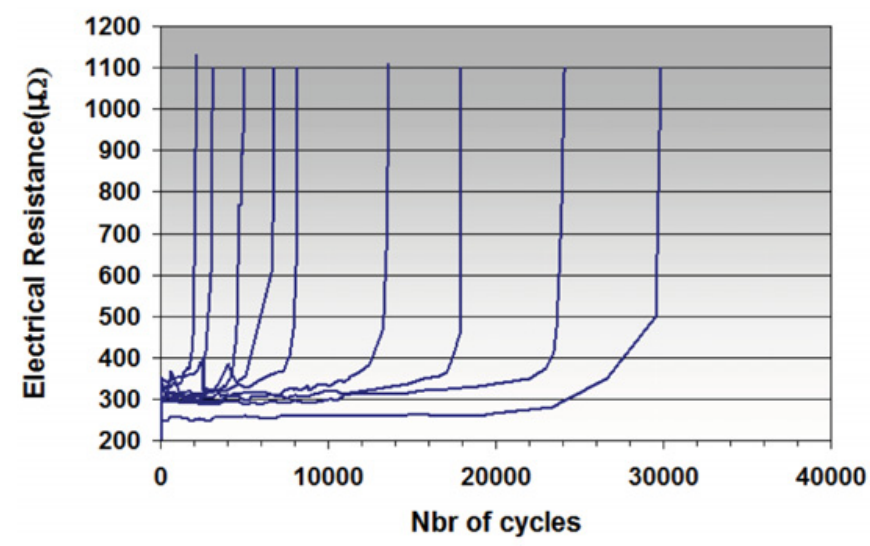

Figure 8. Evolution of the electrical resistance along the cycles (for different values of the test-current).

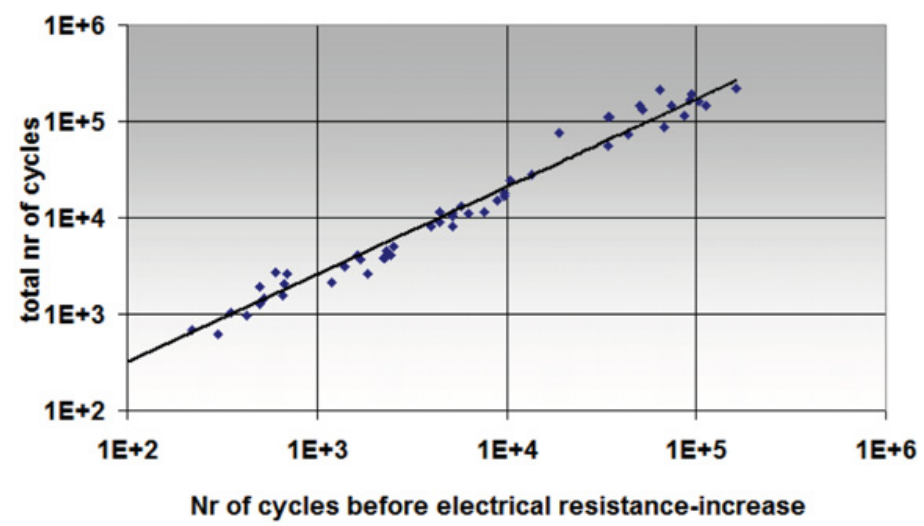

Figure 9. Curve of total number of cycles to rupture vs. number of cycles to crack-opening.

\section{Conclusion}

For people who are not involved neither in their technology nor in their applications, electrical fuses are very simple devices. Anybody has been talked about them as an application of Joule's effect. In fact, they can become a very complex subject when it is about arc-operation and ageing. Generally, fusesmanufacturers and users have an electrical culture and they don't worry so much with materials. It's the reason why they did prefer to build abacus for ageing-calculations. This is regrettable because focusing on materials would bring a better understanding of what occurs and lead to innovative solutions. That's the way that Mersen engaged with the tests presented in this paper.

Another ambition of the author should be to show to mechanicals, materials and fatigue experts that beside their domains, some other engineers could have close problems, that they consider with original approach. 